INGENIERIA DE MATERIALES

\title{
Caracterización y evaluación del tiempo de vulcanización de caucho natural colombiano obtenido de tres variedades clonales de hevea brasiliensis
}

\section{Characterization and evaluation of vulcanization time for colombian natural rubber from three hevea brasiliensis tree clones}

\author{
Sandra M. Velásquez*, Diego H. Giraldo** \\ *Grupo de Investigación BIOMATIC. SENA, Antioquia, COLOMBIA. \\ **Grupo GIPIMME, Facultad de Ingeniería, Universidad de Antioquia, COLOMBIA. \\ *smvelasquez@sena.edu.co**dhernan.giraldo@udea.edu.co
}

Recibido: 08 de agosto de 2013 - Aceptado: 14 de julio de 2014

\begin{abstract}
Resumen
En las últimas décadas se desarrollan en Colombia importantes proyectos productivos de caucho natural, pero aún hay aspectos por estudiar sobre las propiedades de los materiales producidos. En este trabajo se caracteriza mediante FTIR, DSC y TGA caucho natural obtenido de las variedades clonales FX3864, IAN710 e IAN873 predominantes en Tarazá, Antioquia, comparándolo con caucho de Guatemala SGR10 y caucho técnicamente especificado de Caquetá-Colombia. Se obtuvieron compuestos de caucho siguiendo una misma formulación, variando únicamente el tipo de caucho natural base. Las mezclas crudas se evaluaron por reometría de vulcanización a $160^{\circ} \mathrm{C}$ y se moldearon probetas cilíndricas y tipo corbatín en prensa, determinando el tiempo óptimo de vulcanización para cada geometría basada en el módulo elástico bajo compresión uniaxial y bajo tensión, una metodología que puede aplicarse también a productos comerciales. Con los tiempos óptimos de vulcanización encontrados se fabricaron probetas para pruebas de compression-set, densidad y dureza. Se detectaron algunas diferencias entre los espectros FTIR de los cauchos naturales analizados, la temperatura de descomposición, las curvas reométricas y las propiedades mecánicas de los compuestos, por lo cual se plantean opciones para procesar los materiales colombianos con mayor confiabilidad.
\end{abstract}

Palabras clave: Caracterización, caucho natural, caucho técnicamente especificado, vulcanización.

\begin{abstract}
Significant projects for natural rubber (NR) production in Colombia have been developed in the past two decades. However, several topics about the properties of colombian NR have not been studied yet. In this work, NR obtained from FX3864, IAN873 and IAN710 rubber tree clones, cultivated in Tarazá-Antioquia-Colombia, is characterized by FTIR, DSC and TGA. Also, a comparison with SGR10 guatemalan rubber and technically specified rubber of Caquetá, Colombia, is carried out. Rubber compounds were manufactured following the same formulation, varying only the type of natural rubber used as matrix. Uncured mixtures were evaluated by vulcanization rheometry at $160{ }^{\circ} \mathrm{C}$, and cylindrical and tension specimens were molded using compression molding. Optimal vulcanization time for each geometry was determinated based on their elastic modulus under tension and uniaxial compression, a methodology that can be applied to commercial products. Test specimens for compression-set, density and hardness were moulded using the vulcanization optimal times. Some differences between FTIR spectra, decomposition temperature, rheometric parameters and mechanical properties of the natural rubbers compounds were identified. Some recommendations for Colombian natural rubbers processing are postulated, seeking a better reliability.
\end{abstract}

Keywords: Characterization, natural rubber, technically specified rubber, vulcanization. 


\section{Introducción}

La mejora de los árboles hevea brasiliensis para la producción de caucho natural a nivel industrial se logra incorporando nuevas variedades genéticas, y Colombia no es la excepción, buscando especialmente la resistencia a enfermedades como el tizón de la hoja en Suramérica (Sterling \& Rodríguez 2011). En la última década en algunas regiones de Colombia se han implementado cultivos de caucho natural de gran envergadura, dado que constituyen una alternativa socio-económica de alto impacto para las regiones que cultivan y para el sector industrial que lo consume. De acuerdo con las últimas estadísticas consolidadas por el Ministerio de Agricultura colombiano, el área cultivada con caucho en el país ha aumentado de 2.500 hectáreas en 2007 a 7.500 hectáreas en 2011, con una tasa de crecimiento promedio del $27 \%$ (Agronet 2011), por lo cual se requiere avanzar en el conocimiento de los cauchos naturales producidos en las diferentes regiones del país y generar información suficiente para la utilización en procesos industriales de manera confiable. Tres variedades clonales de origen brasileño identificadas como FX 3864, IAN 873 y IAN 710, son cultivadas ampliamente en el país gracias a su resistencia al mal suramericano de la hoja (Sterling \& Rodríguez 2011), sin embargo, sólo se conoce un estudio sobre los cauchos producidos por estos clones en Colombia (Urrego et al. 2012), mientras que para cauchos naturales brasileños o asiáticos se reporta que las características fisicoquímicas del material obtenido dependen de la variedad clonal de origen (Oliviera et al. 2004, Oliviera et al. 2006, Gonçalves 2001, Olviera et al. 2003, Martins et al. 2008, Dall'Antonia et al. 2009). Es bien identificado también que las propiedades mecánicas de los compuestos de caucho vulcanizado dependen de las condiciones de proceso, los componentes de la formulación y los cauchos base (Vergnaud \& Rosca 2010).

El objetivo de este trabajo es identificar las características físicoquímicas de tres tipos de caucho natural producidos en una subregión colombiana, así como la procesabilidad y las propiedades mecánicas de una formulación basada en esos materiales y compararlos con un caucho técnicamente especificado colombiano y uno guatemalteco. También se evalúa una metodología que permita a la industria determinar en la planta de producción los tiempos óptimos de vulcanización de las piezas moldeadas.

\section{Metodología}

\section{Materiales}

Se extrajo látex de 8 árboles de cada una de las tres variedades clonales de hevea brasiliensis estudiadas - FX3864, IAN 873 e IAN 710- cultivados en el valle de Santa Clara, municipio de Tarazá en el Departamento de Antioquia, Colombia. Se seleccionaron árboles de 14 años de edad, 8 años de explotación y beneficiados por el sistema D2, es decir, sangrados cada dos días. El látex fue coagulado durante 24 horas con una solución de agua con ácido acético diluido al 1\% en volumen, luego fue lavado para eliminar el ácido acético residual y así proceder con la laminación en un equipo de rodillos abiertos, para finalmente secar durante 8 días bajo techo pero al aire libre. Para efectos comparativos, se estudió también caucho guatemalteco SGR 10 y caucho técnicamente especificado proveniente de Caquetá - Colombia adquirido en distribuidoras comerciales. Se usó la formulación de la Tabla 1, buscando una dureza de 60 Shore A pretendiendo usar un compuesto industrial.

Tabla 1. Composición de la formulación de Caucho Natural.

\begin{tabular}{cc}
\hline COMPONENTE & CANTIDAD (PHR) \\
\hline Caucho Natural & 100 \\
Sílice granulada & 20 \\
Óxido de Zinc & 5 \\
Ácido esteárico & 3 \\
Negro de Humo 330 & 20 \\
Colofonia & 5 \\
Aceite nafténico medio & 10 \\
Polietilén Glicol & 10 \\
Antioxidante & 1,5 \\
Azufre & 3 \\
TBBS & 1 \\
TMTD & \\
\hline
\end{tabular}

*Sulfenamida N-tert-butyl-2- benzotiazol

**Disulfuro de Tetrametiltiurum 


\section{Procedimiento experimental}

Las láminas de caucho natural se analizaron por espectroscopia de infrarrojo con transformada de Fourier (FTIR), calorimetría diferencial de barrido (DSC) y análisis temogravimetrico (TGA), métodos usados en trabajos sobre la caracterización de caucho natural (Forrest et al. 1997, Brazier 1980). El análisis por FTIR se efectuó en un equipo Perkin Elmer Spectrum One, a $24^{\circ} \mathrm{C}$, en un rango de 4000 a $450 \mathrm{~cm}^{-1}$ aplicando 60 barridos. El análisis por DSC se efectuó en un equipo Q200 V24.2 Build 107 aplicando un borrado térmico hasta $250{ }^{\circ} \mathrm{C}$ y luego enfriando desde temperatura ambiente a una velocidad de $20{ }^{\circ} \mathrm{C} / \mathrm{min}$ hasta $-80{ }^{\circ} \mathrm{C}$ y luego efectuando un análisis por DSC modulado (MDSC) con una señal de $1.27{ }^{\circ} \mathrm{C} / \mathrm{min}$ aplicando un calentamiento de $2{ }^{\circ} \mathrm{C} / \mathrm{min}$ hasta temperatura ambiente. El análisis por TGA se realizó en un equipo Q500 V20.8 Build 34 InstrSerial 05001190, aplicando una velocidad de calentamiento de $20{ }^{\circ} \mathrm{C} /$ min entre 25 y $900{ }^{\circ} \mathrm{C}$.

La mezcla se realizó en un molino de rodillos de tipo abierto a temperatura ambiente inicialmente durante 4 a 5 minutos, incorporando los aditivos diferentes a los acelerantes durante entre 6 y 8 minutos y por último se adicionaron los acelerantes para la homogeneización final entre 2 y 4 minutos, obteniendo láminas con un espesor aproximado de 2,5 a $3 \mathrm{~mm}$. Las mezclas fueron realizadas el mismo día y en las mismas condiciones, buscando variar únicamente el tipo de caucho natural base. De los compuestos crudos se tomaron muestras para efectuar pruebas de reometría de vulcanización a $160^{\circ} \mathrm{C}$ bajo norma ASTM D5289 (ASTM 2007).

Se procedió a determinar los tiempos óptimos de vulcanización de las 5 mezclas para dos geometrías de probeta: cilindros de $29 \mathrm{~mm}$ de diámetro y 12.5 mm de altura para acogerse a la norma ASTM D395 (ASTM 2008), y probetas tipo corbatín para ensayos de tensión según norma ASTM 412 (ASTM 2008). Las probetas se vulcanizaron en moldes a $160^{\circ} \mathrm{C}$ en una prensa de accionamiento hidráulico y calentamiento por resistencias eléctricas, fabricando tres probetas para cada condición de proceso moldeando los cilindros durante 4.5, 5.0, 5.5, 6.0 y 6.5 minutos y las probetas tipo corbatín durante $6.5,7.0,7.5,8.0$ y 8.5 minutos, tiempos que fueron seleccionados de acuerdo a los resultados de las pruebas preliminares.

Las probetas cilíndricas se vulcanizaron en un molde de una sola cavidad, mientras que para obtener las muestras tipo corbatín se utilizó un molde circular con una cavidad de $30 \mathrm{~cm}$ de diámetro y $3.5 \mathrm{~mm}$ de altura, de tal forma que el disco vulcanizado se troquelaba para obtener las probetas con geometría según ASTM 412. Para cada condición de ensayo se fabricaron 3 probetas, seleccionando 2 aleatoriamente para ser sometidas a pruebas mecánicas y almacenando la restante como probeta testigo.

Las probetas cilíndricas se sometieron a compresión uniaxial bajo norma ASTM D575 (ASTM 2007) evaluando el módulo elástico; las probetas tipo corbatín se sometieron a tensión según norma ASTM 412 determinando el módulo al 100\%. Para cada material y cada geometría se determinó el tiempo óptimo de vulcanización, identificando el punto de inflexión en las gráficas módulo elástico versus tiempo de vulcanización.

\section{Determinación de propiedades mecánicas}

Usando el mismo procedimiento de moldeo señalado en la sección anterior, pero empleando el tiempo de vulcanización óptimo que se determinó, se fabricaron para cada material cinco probetas cilíndricas de $12.5 \pm 0.5 \mathrm{~mm}$ de espesor y $29.0 \pm 0.5 \mathrm{~mm}$ de diámetro. Se seleccionaron aleatoriamente tres probetas y se sometieron a ensayos de compresión uniaxial bajo norma ASTM D575 utilizando una máquina universal de ensayos DIGIMESS deformando las probetas $2 \mathrm{~mm}$ a una velocidad de $20 \mathrm{~mm} /$ minuto. Para cada mezcla se moldearon también cinco probetas de tensión y se seleccionaron aleatoriamente tres probetas que se sometieron a tensión bajo norma ASTM 412 en una máquina universal DIGIMESS empleando una velocidad de mordazas de $500 \mathrm{~mm} /$ minuto, alcanzando el desplazamiento máximo que permite la máquina. En los ensayos de tensión se determinó el módulo al $100 \%$ y el módulo al $300 \%$, y a las probetas se les midió dureza bajo norma ASTM D2240 


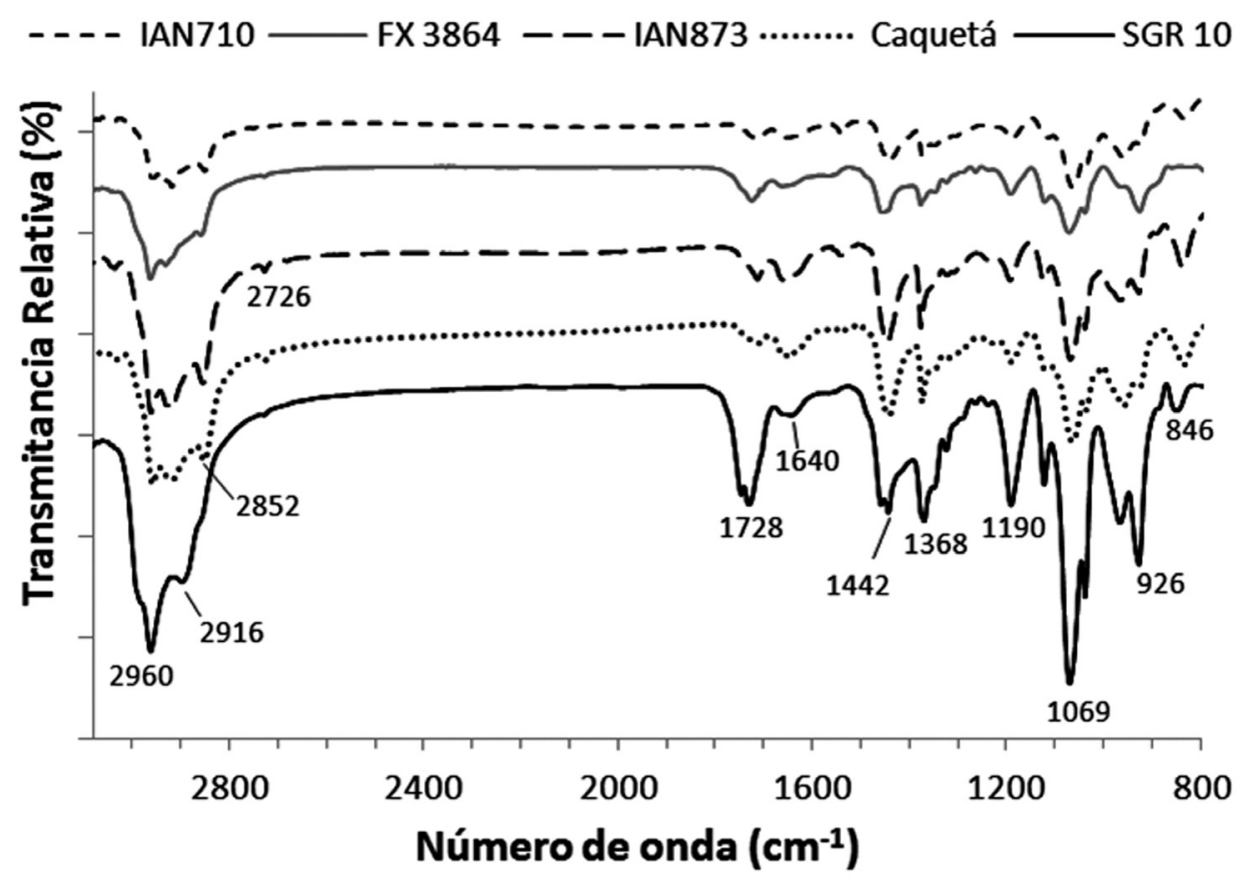

Figura 1. Espectro FTIR de los cauchos naturales estudiados.

(2010) y densidad bajo norma ASTM D792 (2008). Para identificar variaciones debidas al mezclado de los materiales se efectuó un análisis tipo ANOVA con el fin de evaluar si existían diferencias entre las densidades de las probetas cilíndricas y tipo corbatín. Se fabricaron probetas para pruebas de compression-set bajo norma ASTM D395 método B, pruebas efectuadas a $70^{\circ} \mathrm{C}$ por un periodo de 72 horas en un horno de aire circulante marca BINDER.

\section{Resultados y discusión}

Los resultados del análisis por FTIR se muestran en la Figura 1, graficando la transmitancia relativa con el fin de visualizar los espectros con mayor claridad.

Los picos en las regiones próximas a 2850 y 2920 $\mathrm{cm}^{-1}$ corresponden a los estiramientos simétrico y asimétrico del grupo metilo, y en la región de $2960 \mathrm{~cm}^{-1}$ se encuentra el estiramiento simétrico y asimétrico del $\mathrm{C}-\mathrm{H}$ en el grupo funcional $\mathrm{CH}_{3}$. En la región de 1660 y $1544 \mathrm{~cm}^{-1}$ se observa vibración de estiramiento asociado al doble enlace $\mathrm{C}=\mathrm{C}$, en 1444 y $1376 \mathrm{~cm}^{-1}$ vibración de deformación asimétrica y simétrica del C-H en el grupo metilo, en 1126 $\mathrm{cm}^{-1}$ vibraciones del esqueleto $\mathrm{C}-\mathrm{C}$, en $1036 \mathrm{~cm}^{-1}$ deformación axial del enlace $\mathrm{C}-\mathrm{CH}_{2}$ en el plano, en $840 \mathrm{~cm}^{-1}$ un pico fuerte asociado a la flexión del $\mathrm{C}$-H fuera del plano en el grupo funcional $\mathrm{C}=\mathrm{C}$ que se describe como típica de la cadena del cis-1,4 y en $756 \mathrm{~cm}^{-1}$ un pico que corresponde a vibraciones del enlace $\mathrm{C}-\mathrm{C}$ en el grupo funcional $-\left(\mathrm{CH}_{2}\right) \mathrm{x}-$. Las tres variedades clonales cultivadas en Tarazá poseen espectros muy similares a los cauchos técnicamente especificados de Caquetá y SGR 10, con las bandas atribuibles al poli(cis-1,4-isopreno), pero se encuentran diferencias en las intensidades de algunas bandas como 2962, 2918, 2852, 1444, 1376,842 y $756 \mathrm{~cm}^{-1}$ que son representativas del caucho natural, Forrest et al (1997), diferencias que posteriormente serán consideradas para el análisis de los demás resultados obtenidos en el trabajo. Los picos de absorción a 1658 y $1544 \mathrm{~cm}^{-1}$ son más fuertes en el IAN873 y el FX3864 que en los demás materiales, siendo menos fuertes en el IAN710 y con las menores intensidades en los cauchos de Caquetá y el SGR10.

La tabla 2 presenta las temperaturas de transición vítrea (Tg) de los materiales, obtenidos por MDSC, en la cual se puede observar que los resultados son muy similares para los materiales estudiados. 
Tabla 2. Temperaturas asociadas a la transición vitrea (Tg) de los materiales estudiados.

\begin{tabular}{cccc}
\hline Material & Tg inicial $\left({ }^{\circ} \mathbf{C}\right)$ & $\boldsymbol{T g}$ final $\left({ }^{\circ} \mathbf{C}\right)$ & $\operatorname{Tg}\left({ }^{\circ} \mathbf{C}\right)$ \\
\hline FX3864 & -66.65 & -64.86 & -63.01 \\
IAN 710 & -67.45 & -65.45 & -63.80 \\
IAN 873 & -67.08 & -64.98 & -63.24 \\
Caquetá & -67.12 & -63.34 & -64.98 \\
SGR10 & -66.85 & -64.92 & -63.07 \\
\hline
\end{tabular}

La figura 2 muestra las curvas de TGA obtenidas, encontrando que la descomposición comienza aproximadamente a $300{ }^{\circ} \mathrm{C}$ y termina a temperaturas próximas a los $550{ }^{\circ} \mathrm{C}$ perdiendo en el proceso alrededor del $99 \%$ de la masa, observándose el hombro típico de los cauchos naturales, Brazier (1980).

El IAN710 mostró una temperatura de descomposición térmica significativamente más baja que los demás materiales, y el IAN873 y el FX3864 muestran unas temperaturas de descomposición más alta que los demás materiales. Al terminar el análisis se encontraron residuos entre $0.2 \%$ y $0.6 \%$ en peso, siendo el contenido más bajo para el IAN710 $(0.22 \%)$ y el contenido más alto para el FX3864 (0.60 \%). El residuo obtenido después de la degradación termo-oxidativa a 550
${ }^{\circ} \mathrm{C}$ se asocia a cenizas comunes en el látex al salir de la corteza o impurezas que contaminaron el látex durante la extracción y coagulación en la plantación.

Se obtuvieron las mezclas crudas y se determinó su densidad, obteniendo valores entre 1.09 y 1.11 $\mathrm{gr} / \mathrm{cm}^{3}$ sin que se observara efecto de la variedad clonal; el coeficiente de variación máximo encontrado al considerar cada mezcla fue de $0.5 \%$, y un análisis ANOVA permitió confirmar que el tiempo de vulcanización no tuvo efecto en la densidad y por lo tanto la variabilidad inherente al proceso de pesaje de los componentes de formulación y masticación en molino no generó una diferencia estadísticamente significativa entre los componentes de las mezclas.

La figura 3 muestra los resultados de las pruebas de reometría de vulcanización a las 5 mezclas.

Se observan diferencias significativas entre las reometrías de las mezclas, encontrando que la formulación con IAN 710 presentó el mejor tiempo de procesabilidad y el mayor torque, es decir, el mejor resultado. La mezcla con SGR 10 presenta el segundo mejor tiempo de proceso, pero con un torque máximo similar a la mezcla con FX3864,

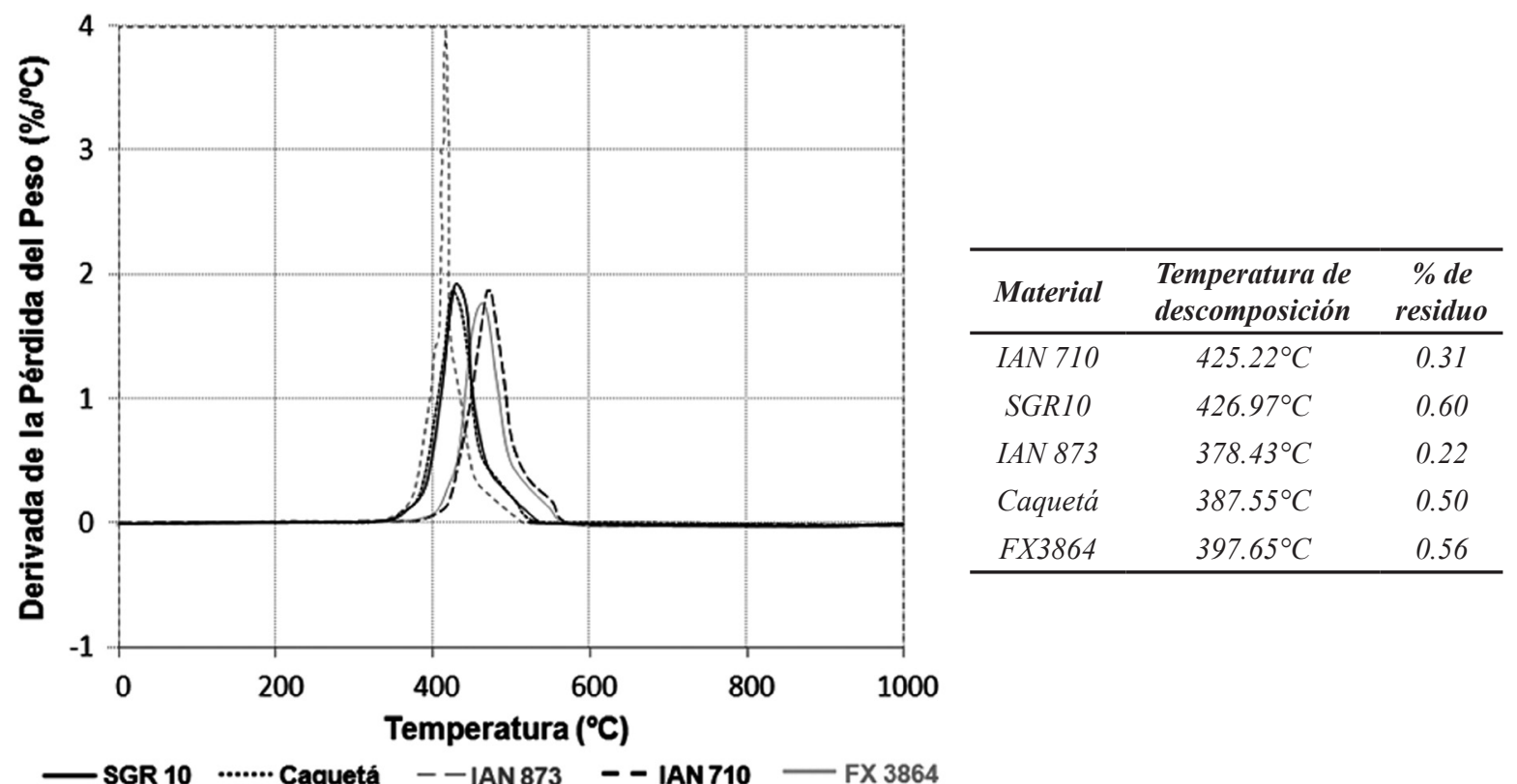

Figura 2. TGA comparativo de los cinco caucho naturales. 


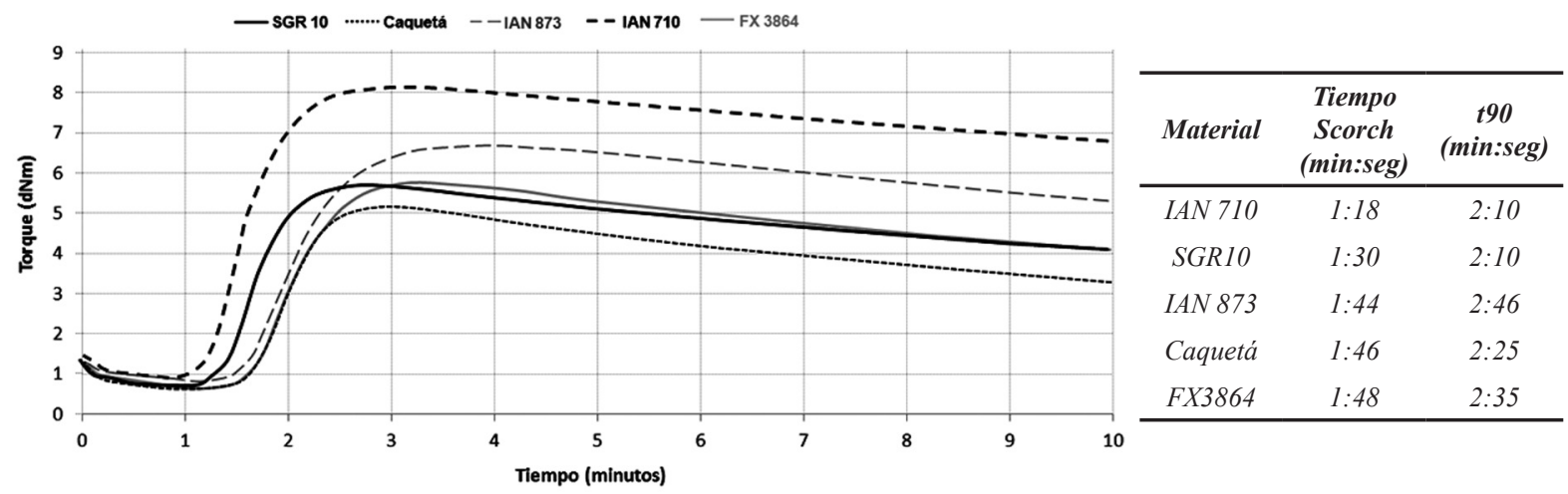

Figura 3. Curvas reométricas comparativas de las cinco mezclas efectuadas con los cauchos naturales estudiados.

siendo ambos inferiores al de la mezcla con IAN 873. La mezcla con material de Caquetá presenta un tiempo scorch y 190 ligeramente inferiores a los de la mezcla con FX3864, pero con el menor torque de todas las mezclas. En todos los casos se observó la reversión típica del caucho natural.

Los tiempos óptimos de vulcanización para las geometrías cilíndrica y tipo corbatín se determinaron evaluando dureza, propiedades determinadas en pruebas de tensión y compresión uniaxial. El efecto del tiempo de vulcanización en la dureza de ambos tipos de geometría se muestra en la figura 4, y la variación del módulo elástico bajo compresión y del módulo al $100 \%$ con el tiempo de vulcanización se muestra en la figura 5.

Las curvas presentan un máximo al variar el tiempo de vulcanización, lo cual se asocia a la reversión del caucho natural. Los tiempos óptimos de vulcanización varían según la mezcla, recordando que el módulo elástico bajo compresión uniaxial y el módulo al 100\% en tensión son más representativos de los compuestos vulcanizados que la dureza Shore A, que toma mediciones localizadas de carácter superficial aunque es una prueba de amplia aplicación industrial dada su fácil realización en planta, como control de calidad. En las probetas cilíndricas se observó que los menores tiempos de vulcanización corresponden al SGR 10 y al caucho de Caquetá, seguido por el IAN 873; las mezclas con IAN 710 y FX 3864 presentan el mayor tiempo óptimo de vulcanización. En las probetas tipo corbatín los menores tiempos óptimos de vulcanización se encontraron para el SGR 10, el IAN 873 y el FX3864, y los mayores tiempos se observaron para las mezclas con IAN 710 y material del Caquetá.

Con las condiciones óptimas de vulcanización para cada mezcla se procedió a fabricar las probetas para pruebas de tensión, compresión y compression-set. En la figura 6 se muestran los resultados de estas pruebas.
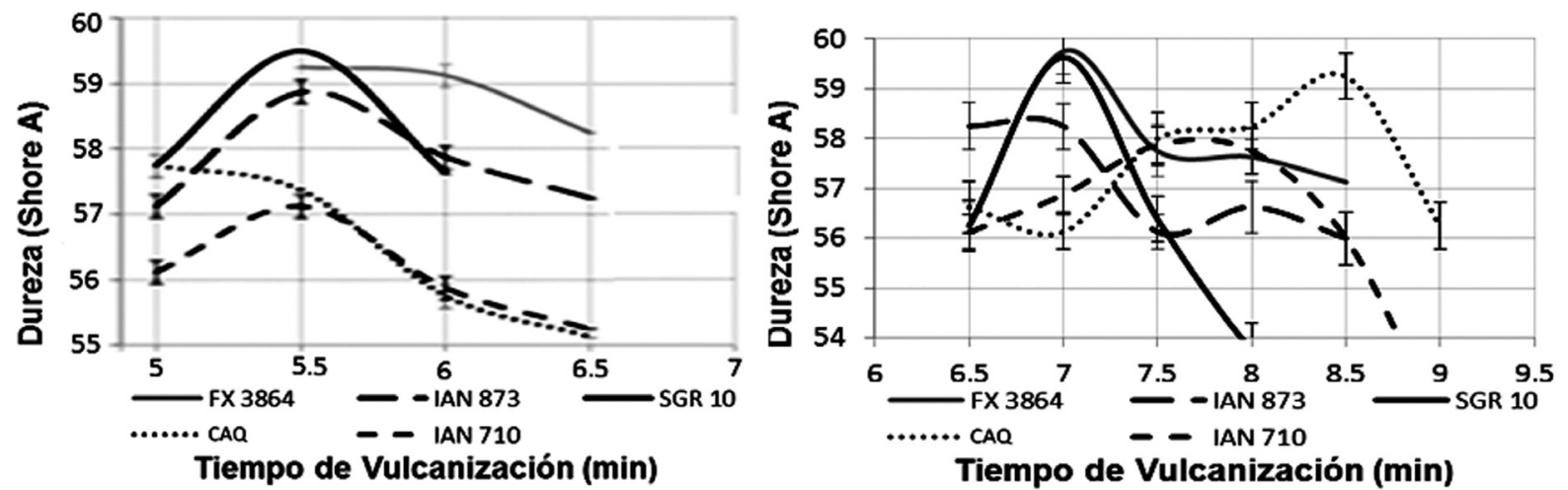

Figura 4. Curvas dureza vs tiempo de vulcanización para las probetas cilíndricas (izquierda) y tipo corbatín (derecha) para las mezclas estudiadas ya vulcanizadas. 


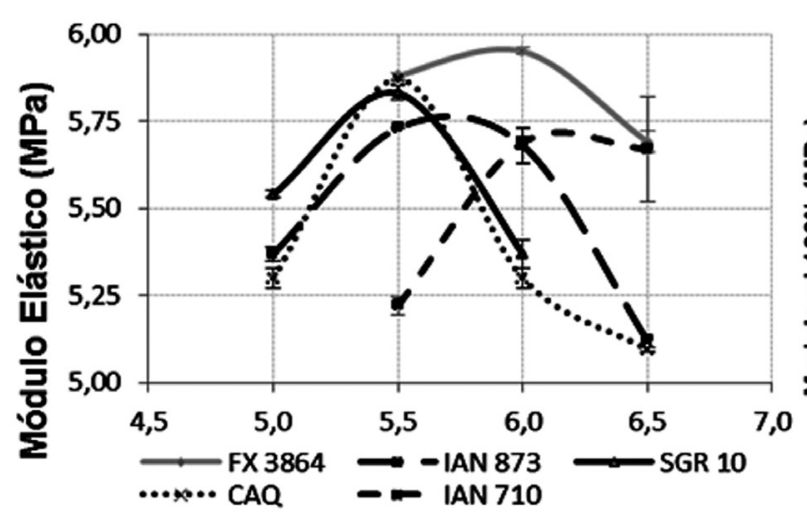

Tiempo de Vulcanización (min)

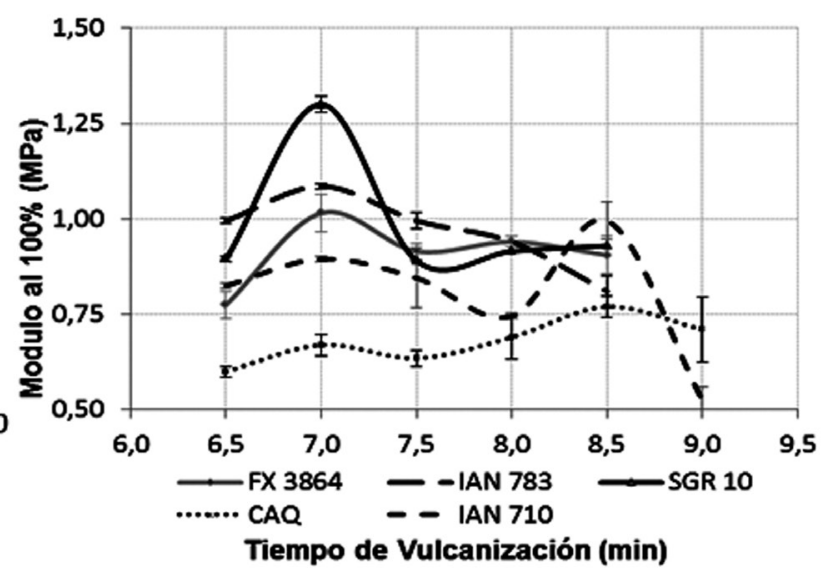

Figura 5. Módulo elástico bajo compresión uniaxial (izquierda) y módulo al 100\% en tensión (derecha) de las mezclas versus el tiempo de vulcanización.
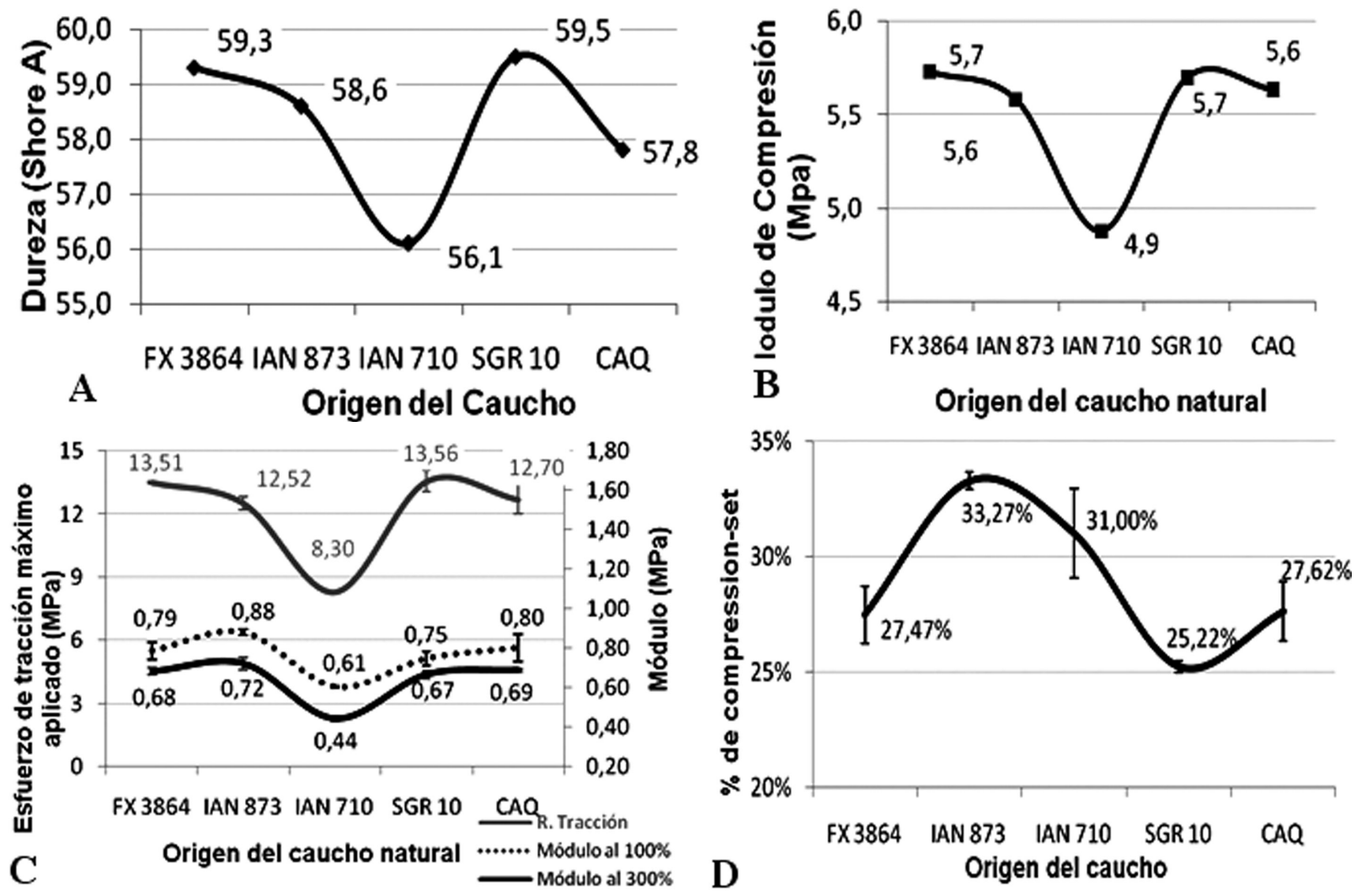

Figura 6. Valores de dureza (A), módulo de compresion (B), resistencia a tensión y módulos a tensión (C) y compressión set (D) para las diferentes variedades clonales de caucho natural en su tiempo óptimo de vulcanización.

La mezcla con IAN710 muestra las menores propiedades mecánicas de los materiales estudiados, mientras que las propiedades de las mezclas con SGR10 y FX3864 son superiores a las de los demás materiales, y son muy similares entre sí. Las mezclas con cauchos del Caquetá e IAN873 presentan propiedades intermedias y muy similares entre sí. El caucho obtenido con SGR10 presentó el menor compression-set, el material de Caquetá y el FX3864 valores superiores y los materiales con la peor respuesta a deformaciones permanentes son el IAN873 y el IAN710. 


\section{Conclusiones}

Los resultados encontrados indican que existen diferencias representativas entre las propiedades mecánicas de los materiales analizados, observando que el caucho proveniente de la variedad clonal FX3864 presenta propiedades superiores a las otras dos variedades clonales cultivadas en el Valle de Santa Clara, alcanzando valores muy similares a los del caucho técnicamente especificado SGR10 proveniente de Caquetá. En cuanto a la procesabilidad de la mezcla determinada por reometría de vulcanización y por la determinación de los tiempos óptimos de vulcanización, también se detectan diferencias apreciables entre los compuestos, aspecto que sumado a la diferencia en propiedades mecánicas debe ser considerado por los cultivadores al momento de seleccionar las variedades clonales a explotar en la región, así como por la industria cauchera con miras a estandarizar sus procesos de producción.

El análisis por FTIR y por MDSC no permitió asociar las diferencias en propiedades encontradas a diferencias entre los espectros y la temperatura de transición vítrea, por lo cual se plantea efectuar en el futuro cercano un estudio detallado al respecto. El análisis por TGA demostró que el caucho proveniente de la variedad clonal IAN 710 presenta la menor temperatura de descomposición, mientras que los cauchos de las variedades FX3864 e IAN 873 presentan las mayores temperaturas de descomposición entre los cauchos estudiados, una demostración más de las diferencias entre propiedades de estos materiales.

Los cultivadores de caucho natural en Colombia deben efectuar modificaciones al método de selección de las variedades clonales a cultivar, dado que en los resultados de este trabajo han demostrado que las características de los materiales obtenidos son sensibles al tipo de clon de origen del caucho natural. En la región de estudio el caucho proveniente de la variedad clonal FX3864 demostró poseer las mejores propiedades mecánicas y la mejor estabilidad térmica, superando a los otros dos materiales, mientras que el IAN 710 demostró poseer propiedades significativamente más bajas. Deben efectuarse nuevos estudios que evalúen el efecto de las condiciones climáticas como temperatura ambiente y humedad en las propiedades de los cauchos naturales producidos, tal y como lo han realizado investigadores del área del caucho principalmente de países con gran tradición cauchera.

Se planteó una metodología para la determinación de tiempos óptimos de vulcanización de mezclas de caucho que puede ser utilizada en piezas vulcanizadas en plantas de producción, como una alternativa a la práctica más tradicional de determinar la dureza Shore A, metodología que demostró ser sensible al tipo de caucho natural usado en cada formulación.

La industria cauchera, en este caso la colombiana, debe estar permanentemente atenta a las propiedades de los cauchos naturales que usan como materia prima dado que las características de este tipo de materiales dependen del tipo de variedad clonal de origen, evidenciando la necesidad de fortalecer el trabajo conjunto con los cultivadores de la región de tal manera que ambos sectores se ven beneficiados mutuamente mediante el mejoramiento de sus procesos.

\section{Referencias bibliográficas}

AGRONET \& SEA (Red de Información y Comunicación Estratégica del Sector Agropecuario \& Sistema de Estadísticas Agropecuarias). (2011). Producción de Caucho en Colombia, 2007 2011. http://www.agronet.gov.co/www/htm 3 b/ ReportesAjax/VerReporte.aspx.

ASTM D5289 - 07a. (2007) Standard Test Method for Rubber Property-Vulcanization Using Rotorless Cure Meters.

ASTM D395 -03. (2008). Standard Test Methods for Rubber Property - Compression Set.

ASTM D412. (2008). Standard Test Methods for Vulcanized Rubber and Thermoplastic Elastomers-Tension. 
ASTM D575 - 91. (2007). Standard Test Methods for Rubber Properties in Compression.

ASTM D2240 - 05. (2010). Standard Test Method for Rubber Property—Durometer Hardness.

ASTM D792 - 08. (2008). Standard Test Methods for Density and Specific Gravity (Relative Density) of Plastics by Displacement.

Brazier, D. W. (1980). Applications of thermal analytical procedures in the study of elastomers and elastomer system. Rubber Chem. Technol. 53, 437-511.

Dall'Antonia, A.C., Martins, M. A., Moreno, R., Goncalves, P., Mattoso, L.H. \& Job, A.E. (2009). Caracterização Mecânica e Térmica da Borracha Natural Formulada e Vulcanizada dos Clones: GT 1, IAN 873, PB 235 e RRIM 600. Ciência e Tecnologia 19(1), 63-71.

Forrest, M., Davies, Y., \& Davies, J. (1997). The RAPRA collection of infrared spectra of rubbers practics and thermoplastic elastomers, 2nd editión, by Rapra technology limited.

Goncalves, P.S., Bortoletto, N., Furtado, E.L., Sambugaro, O.C. \& Bataglia, A.A. (2001). Desempenho de clones de seringueira da série IAC300 selecionados para a região noroeste do estado de São Paulo, Pesquisa Agropecuária Brasileira (Brasilia) 36 (4), 589-598.

Martins, M. A., Moreno, R., Colleen M., McMahan, C.M.,. Brichta, J.L., Gonçalves, P. \& Mattoso, L.H. (2008). Thermooxidative study of raw natural rubber from Brazilian IAC 300 series clones. ELSEVIER. Thermochimica Acta 474. 62-66.

Oliviera, L. C. S., Arruda, E. J., Costa, R. B., Gonçalves, P. S. \& Delben, A. (2003). Evaluation of latex from five Hevea clones grown in São Paulo State, Brazil. ELSEVIER. Thermochimica Acta 398. 259-263.

Oliviera, L. C. S., Arruda, E. J., Favaro, S.P., Costa, R. B., Gonçalves, P. S. \& Job, A.E. (2006). Evaluation of thermal behavior of latex membranes from genetically improved rubber tree (Hevea brasiliensis). Thermochimica Acta 445. 27-31.

Oliviera, L. C. S., Rosa, D. P., Arruda, E. J., Costa, R. B., Gonçalves, P. S. \& Delben, A. (2004). Comparative studies of latex obtained of rubber tree clones (Hevea Brasiliensis) - Series IAC 328 Votuporanga - sp. Journal of Thermal Analysis and Calorimetry 75, 495-500.

Sterling,A.\& Rodríguez, C.H. (2011). Nuevos clones de caucho natural para la Amazonia colombiana: énfasis en la resistencia al mal suramericano de las hojas (Microcyclus ulei). Instituto Amazónico de Investigaciones Cientificas - Sinchi, 196.

Urrego, W., Giraldo. D., Álvarez \& Láinez, M. (2012). Análisis cuantitativo por FTIR y evaluación comparativa de la descomposición térmica de tres variedades de caucho natural colombiano. XIII Simposio Latinoamericano de Polímeros SLAP y XI Congreso Iberoamericano de Polímeros. Bogotá, Colombia.

Vergnaud, J.M. \&Rosca, I. D. (2010). Rubber curing and properties, CRC Press, Boca Raton-USA. 249. 